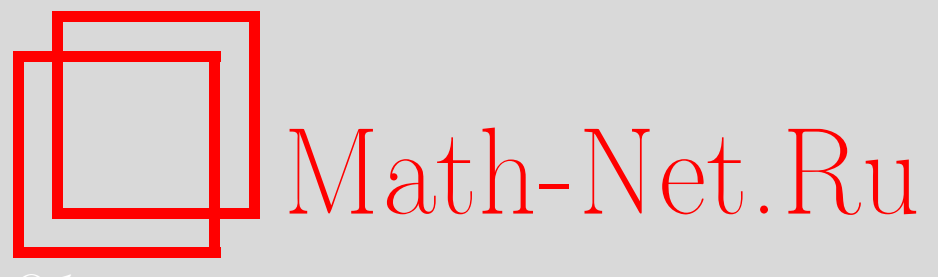

А. А. Фещук, K вопросу анализа нечетких информационных графов, Дискрет. матем., 2002, том 14, выпуск 2, 6584

DOI: https://doi.org/10.4213/dm242

Использование Общероссийского математического портала Math-Net.Ru подразумевает, что вы прочитали и согласны с пользовательским соглашением http://www.mathnet.ru/rus/agreement

Параметры загрузки:

IP : 52.90 .164 .192

26 апреля 2023 г., 10:40:03

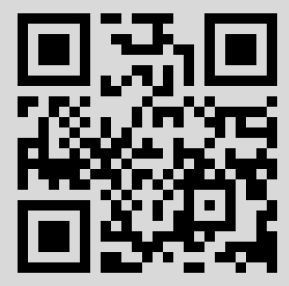




\title{
К вопросу анализа нечетких информационных графов
}

\author{
() 2002 г. A. А. Фещук
}

\begin{abstract}
Предлагается математическая модель для описания информационного поиска с нечеткой логикой. Приводятся простейшие свойства модели такие, как критерий допустимости информационного графа и критерий полноты базового множества. Описывается некоторый метод перехода от четкой задачи поиска к нечеткой и приводятся условия, при которых решение четкой задачи будет решением соответствующей нечеткой.

Работа выполнена при поддержке Российского фонда фундаментальных исследований, проект 01-01-00748.
\end{abstract}

\section{1. Введение}

В работе предлагается математическая модель, предназначенная для описания информационного поиска с нечеткой логикой. Модель вводится как обобщение информационно-графовой модели данных [1]. Полученные простейшие свойства предлагаемой модели такие, как критерий допустимости нечетких информационных графов и критерий полноты базового множества, полностью аналогичны соответствующим свойствам информационно-графовой модели.

Описывается способ перехода от четкой задачи информационного поиска к нечеткой путем размывания отношения поиска и базовых предикатных функций. Размывание этих объектов осуществляется с помощью специальных пикообразных функций, образующих основание перехода. Получен критерий того, когда основание перехода обеспечивает такой переход, при котором произвольный информационный граф, решающий четкую задачу поиска, переходит в нечеткий информационный граф, решающий соответствующую нечеткую задачу.

Автор выражает глубокую благодарность Э. Э. Гасанову за постановку задачи и помощь в работе.

\section{2. Основные понятия и формулировка результатов}

В соответствии с [1] введем понятие задачи информационного поиска. Пусть $X-$ множество запросов; $Y$ - множество записей (объектов поиска); $\rho$ - бинарное отношение на $X \times Y$, называемое отношением поиска; тройку $S=\langle X, Y, \rho\rangle$ будем называть типом; тройку $I=\langle X, V, \rho\rangle$, где $V$ - некоторое конечное подмножество множества $Y$, будем называть задачей информационного поиска (ЗИП) типа $S$ и будем считать, что ЗИП $I=\langle X, V, \rho\rangle$

3 Дискретная математика, т.14 №2 
содержательно состоит в перечисления для произвольно взятого запроса $x \in X$ всех тех и только тех записей $y \in V$, для которых $x \rho y$.

По аналогии с этим определением введем понятие задачи нечеткого поиска. Как и ранее, пусть $X$ - множество запросов, $Y$ - множество записей. Пусть задано отображение $\eta(x, y): X \times Y \rightarrow[0,1]$, которое будем называть отношением нечеткого поиска. Тройку $S=\langle X, Y, \eta\rangle$ будем называть типом нечеткого поиска; тройку $I=\langle X, V, \eta\rangle$, где $V-$ некоторое конечное подмножество множества $Y$, будем называть задачей нечеткого поиска (ЗНП) типа $S$ и будем считать, что ЗНП $I=\langle X, V, \eta\rangle$ содержательно состоит в том, чтобы для произвольного числа $c \in(0,1]$ и произвольного запроса $x \in X$ перечислить все те и только те записи $y \in V$, для которых $\eta(x, y) \geqslant c$.

Введем несколько упрощенное по сравнению с [1] понятие информационного графа.

Пусть $F$ - множество символов одноместных предикатов, определенных на множестве $X$, называемое базовым множеством.

Понятие информационного графа (ИГ) над базовым множеством $F$ определяется следующим образом. Берется конечная многополюсная ориентированная сеть. В ней выбирается некоторый гюлюс, который называется корнем. Остальные полюсы называются листьями, и им приписываются записи из $Y$, причем разным листьям могут быть приписаны одинаковые записи. Ребрам приписываются предикаты из множества $F$. Таким образом нагруженную многополюсную ориентированную сеть называем ИГ над базовым множеством $F$.

Функционирование ИГ определяется следующим образом. Скажем, что ребро проводит запрос $x \in X$, если предикат, приписанный этому ребру, принимает значение 1 на запросе $x$; ориентированная цепочка ребер проводит запрос $x \in X$, если каждое ребро цепочки проводит запрос $x$; запрос $x \in X$ проходит в вершину $\beta$ ИГ, если существует ориентированная цепочка, ведущая из корня в вершину $\beta$, которая проводит запрос $x$; запись $y$, приписанная листу $\alpha$, попадает в ответ ИГ на запрос $x \in X$, если запрос $x$ проходит в лист $\alpha$. Ответом ИГ $U$ на запрос $x$ назовем множество записей, попавших в ответ ИГ на запрос $x$, и обозначим его $\mathscr{I}_{U}(x)$. Эту функцию $\mathscr{L}_{U}(x)$ будем считать результатом функщионирования ИГ $U$.

Пусть дана ЗИП $I=\langle X, V, \rho\rangle$. Скажем, что ИГ $U$ допустим для ЗИП $I=\langle X, V, \rho\rangle$, если $\mathscr{L}_{U}(x)=\{y \in V: x \rho y\}$.

Множество ИГ над базовым множеством $F$, допустимых для ЗИП $I$, обозначим U(I,F).

Аналогично введем понятие нечеткого информационного графа.

Пусть $\mathscr{F}_{F}=\left\{f_{a} \mid f_{a}: X \rightarrow[0,1], a \in A\right\}-$ базовое множество нечетких функций на $X$, где $A$ - некоторое множество индексов.

Понятие нечеткого информационного графа (НИГ) над базовым множеством $\mathscr{F}$, определяется следующим образом. Берется конечная многополюсная ориентированная сеть. В ней выбирается некоторый полюс, который называется корнем. Остальные полюсы называются листьями, и им приписываются записи из $Y$, причем разным листьям могут быть приписаны одинаковые записи. Ребрам приписываются функщии из множества F्F. Таким образом нагруженную многополюсную ориентированную сеть называем НИГ над базовым множеством $\mathscr{F}$.

Функщионирование НИГ определяется следующим образом. Проводимостью ребра НИГ назовем функцию, равную функции, приписанной этому ребру; проводимостью ориентированной цепочки ребер НИГ назовем функщию, равную минимуму проводимостей ребер цепочки; функцией фильтра вершины $\beta$ НИГ назовем функцию, равную максимуму функций проводимости ориентированных цепочек, ведущих из корня НИГ в вершину $\beta$, 
и будем обозначать ее $\varphi_{\beta}(x)$; скажем, что запись $y$, приписанная листу $\alpha$, попадает в ответ НИГ на запрос $x \in X$ для числа $c \in(0,1]$, если $\varphi_{\alpha}(x) \geqslant c$. Ответом НИГ $U$ на запрос $x$ для числа $c \in(0,1]$ назовем множество записей, попавших в ответ НИГ на запрос $x$ для числа $c \in(0,1]$, и обозначим его $\mathscr{I}_{U}(x, c)$. Эту функцию $\mathscr{F}_{U}(x, c)$ будем считать результатом функционирования НИГ $U$.

Скажем, что НИГ $U$ допустим для ЗНП $I=\langle X, V, \eta\rangle$, если для любого запроса $x \in X$ и любого числа $c \in(0,1]$

$$
\mathscr{S}_{U}(x, c)=\{y \in V: \eta(x, y) \geqslant c\} .
$$

Множество НИГ над базовым множеством $\mathscr{F}$, допустимых для ЗНП $I$, обозначим U(I, F्F $)$.

Для $y \in Y$ множество $O(y, \eta)=\{x \in X: \eta(x, y)>0\}$ назовем тенью записи $y$.

Пусть $U$ - некоторый НИГ, $y$ - запись из $Y$. Через $L_{U}(y)$ обозначим множество листьев НИГ $U$, которым соответствует запись $y$.

Справедлива следующая теорема.

Теорема 1. НИГ U допустим для ЗНП $I=\langle X, V, \eta\rangle$ тогда и только тогда, когда

(1) для любой записи $y \in V$ такой, что $O(y, \eta) \neq \varnothing$,

$$
L_{U}(y) \neq \varnothing,
$$

$u$

$$
\max _{\alpha \in L_{U}(y)} \varphi_{\alpha}(x) \equiv \eta(x, y)
$$

(2) для любой записи у $\in V$ такой, что $O(y, \rho)=\varnothing$, либо $L_{U}(y)=\varnothing$, либо

$$
\max _{\alpha \in L_{U}(y)} \varphi_{\alpha}(x) \equiv \eta(x, y) \equiv 0 .
$$

Базовое множество $\mathscr{F}$ называется полным для типа $S=\langle X, Y, \eta\rangle$, если для любой ЗНП $I=\langle X, V, \eta\rangle$ типа $S$ существует НИГ $U$ над базовым множеством $\mathscr{F}$, допустимый для उНП $I$.

Справедлив следующий результат, относящийся к проблеме полноты для НИГ.

Теорема 2. Базовое множество F्ғ является полным для типа $S=\langle X, Y, \eta\rangle$ тогда и только тогда, когда для любой записи $y \in Y$ такой, что $O(y, \eta) \neq \varnothing$, функция $\eta(x, y)$ как функция от х может быть представлена формулой вида

$$
\eta(x, y)=\max _{1 \leqslant i \leqslant n} \min _{1 \leqslant j \leqslant m_{i}} f_{i j}(x),
$$

гдe $f_{i j} \in \mathscr{F}$.

Теоремы 1 и 2 являются обобщениями теорем 1 и 2 из [2].

Далее всюду будем считать, что множество запросов $X=[0,1]$ и множество записей $Y=[0,1]$.

Введем некоторый способ перехода от задачи информационного поиска к задаче нечеткого поиска такой, что для замкнутого $\rho$ и числа $c=1$ задача нечеткого поиска совпадает с исходной задачей информационного поиска. 
Рассмотрим некоторую функцию $\mu: \mathbf{R}^{2} \rightarrow[0,1]$ такую, что

$$
\mu(x, y)=1 \Longleftrightarrow(x, y)=(0,0)
$$

и для любых $x \in[-1,1], y \in[-1,1]$ и любого $\lambda \in(0,1)$ выполняется неравенство $\mu(\lambda x, 0)>\mu(x, 0)$, и если $y \neq 0$, то $\mu(\lambda x, \lambda y) \geqslant \mu(x, y)$. Рассмотрим некоторую функцию $\mu^{\prime}: \mathbf{R} \rightarrow[0,1]$ такую, что $\mu^{\prime}(x)=1 \Leftrightarrow x=0$ и для любого $x \in[-1,1]$ и любого $\lambda \in(0,1)$ выполняется неравенство $\mu^{\prime}(\lambda x)>\mu^{\prime}(x)$. Пару $\phi=\left\langle\mu, \mu^{\prime}\right\rangle$ назовем основанием перехода. Основание $\phi=\left\langle\mu, \mu^{\prime}\right\rangle$ позволяет задать оператор перехода $\phi$, который каждой ЗИП позволяет сопоставить некоторую ЗНП, а каждому ИГ некоторый НИГ.

Пусть $\rho \subseteq[0,1]^{2}-$ отношение поиска. Через $\phi(\rho)$ обозначим нечеткое отношение

$$
\eta(x, y)=\sup _{\left(x_{0}, y_{0}\right) \in \rho} \mu\left(x-x_{0}, y-y_{0}\right)
$$

Заметим, что если $\rho \neq \varnothing$, то множество $\left\{(x, y) \in[0,1]^{2}: \eta(x, y)=1\right\}$ замкнуто.

Каждой ЗИП $I=\langle[0,1], V, \rho\rangle$ сопоставим ЗНП $\phi(I)=\langle[0,1], V, \phi(\rho)\rangle$.

Пусть $F$ - некоторое базовое множество предикатов на $[0,1]$. Для $f \in F$ пусть

$$
N_{f}=\{x \in[0,1]: f(x)=1\},
$$

и для $A \subseteq[0,1]$ пусть

$$
\mu^{\prime}(A)(x)=\sup _{x_{0} \in A} \mu^{\prime}\left(x-x_{0}\right)
$$

Піредикату $f \in F$ сопоставим функцию $\phi(f)(x)=\mu^{\prime}\left(N_{f}\right)(x)$. Множеству $F$ сопоставим базовое множество нечетких функций $\phi(F)=\{\phi(f): f \in F\}$. Заметим, что если $N_{f} \neq \varnothing$, то множество $\{x \in[0,1]: \phi(f)(x)=1\}$ замкнуто.

Пусть $U-$ ИГ над базовым множеством $F$. Сопоставим ему НИГ $\phi(U)$ над базовым множеством $\phi(F)$, полученный из ИГ $U$ заменой для каждого ребра ИГ $U$ предиката $f$, приписанного этому ребру, на функцию $\phi(f)$.

Для произвольного базового множества предикатов $F$ рассмотрим всевозможные конечные подмножества $F_{\lambda} \subseteq F$. Для каждого $F_{\lambda}$ и произвольного основания перехода $\phi=\left\langle\mu, \mu^{\prime}\right\rangle$ рассмотрим функцию $g_{\lambda, \phi}(x)=\min _{f \in F_{\lambda}} \phi(f)(x)$. Заметим, что если $g_{\lambda, \phi}(x)<1$ для всех $F_{\lambda} \subseteq F$ и всех $x \in[0,1]$, то либо базовое множество $F$ пусто, либо $N_{f}=\varnothing$ для всех предикатов $f \in F$

Учитывая, что в дальнейшем основание перехода будет предполагаться фиксированным, символ $\phi$ в обозначении $g_{\lambda, \phi}$ будем опускать, то есть положим $g_{\lambda}(x) \equiv g_{\lambda, \phi}(x)$ при выбранном $\phi$.

Фиксируем основание перехода $\phi$. Для тех индексов $\lambda$, для которых функция $g_{\lambda}$ принимает значение 1 , положим

$$
a_{\lambda}^{\prime}=\sup _{\substack{0 \leqslant x \leqslant 1 \\ \forall t \in[0, x] g_{\lambda}(t)=\mu^{\prime}(t-x)}} x, \quad a_{\lambda}^{\prime \prime}=\sup _{\substack{0 \leqslant x \leqslant 1 \\ \forall t \in[x, 1] g_{\lambda}(t)=\mu^{\prime}(t-x)}}(1-x),
$$

Если $g_{\lambda}(x)<1$ для всех $x \in[0,1]$, то положим $a_{\lambda}^{\prime}=a_{\lambda}^{\prime \prime}=b_{\lambda}^{\prime}=b_{\lambda}^{\prime \prime}=0$. 
Положим

$$
\begin{array}{ll}
a_{F}^{\prime}=\sup _{\lambda} a_{\lambda}^{\prime}, & a_{F}^{\prime \prime}=\sup _{\lambda} a_{\lambda}^{\prime \prime}, \\
b_{F}^{\prime}=\sup _{\lambda} b_{\lambda}^{\prime}, & b_{F}^{\prime \prime}=\sup _{\lambda} b_{\lambda}^{\prime \prime} .
\end{array}
$$

Заметим, что $a_{F}^{\prime} \leqslant b_{F}^{\prime}, a_{F}^{\prime \prime} \leqslant b_{F}^{\prime \prime}$.

Положим $u_{F}=1$, если $b_{F}^{\prime}=b_{\lambda}^{\prime}$ или $b_{F}^{\prime \prime}=b_{\lambda}^{\prime \prime}$ для некоторого $\lambda$. Если такого $\lambda$ не существует, то положим $u_{F}=0$. Если $a_{F}^{\prime}=b_{F}^{\prime}$ и $a_{F}^{\prime}=a_{\lambda}^{\prime}$ для некоторого $\lambda$, то положим $u_{F}^{\prime}=1$, в противном случае положим $u_{F}^{\prime}=0$. Если $a_{F}^{\prime \prime}=b_{F}^{\prime \prime}$ и $a_{F}^{\prime \prime}=a_{\lambda}^{\prime \prime}$ для некоторого $\lambda$, то положим $u_{F}^{\prime \prime}=1$, в противном случае положим $u_{F}^{\prime \prime}=0$.

Для каждого подмножества $F_{\lambda} \subseteq F$, для которого $g_{\lambda}$ принимает значение 1 , рассмотрим множество точек $V_{\lambda}=\left\{v_{1}^{\lambda}, v_{2}^{\lambda}, \ldots\right\}$ таких, что $g_{\lambda}\left(v_{i}^{\lambda}\right)<1$ и существует $x \in[0,1]$, для которого $g_{\lambda}(x)=1$ и либо для всех $t \in\left[x, v_{i}^{\lambda}\right)$

$$
g_{\lambda}(t)=\mu^{\prime}(t-x)
$$

и в некоторой окрестности $v_{i}^{\lambda}$ при $t>v_{i}^{\lambda}$ выполняется неравенство $g_{\lambda}(t) \neq \mu^{\prime}(t-x)$, либо для всех $t \in\left(v_{i}^{\lambda}, x\right]$

$$
g_{\lambda}(t)=\mu^{\prime}(t-x)
$$

и в некоторой окрестности $v_{i}^{\lambda}$ при $t<v_{i}^{\lambda}$ выполняется неравенство $g_{\lambda}(t) \neq \mu^{\prime}(t-x)$. Обозначим $c_{i}^{\lambda}=g_{\lambda}\left(v_{i}^{\lambda}\right)$. Если $0 \in V_{\lambda}$ для некоторого индекса $\lambda$, то положим

$$
b^{\prime}=\inf _{\lambda, i: v_{i}^{\lambda}=0} c_{i}^{\lambda}
$$

в противном случае положим $b^{\prime}=\infty$. Если $1 \in V_{\lambda}$ для некоторого индекса $\lambda$, то положим

$$
b^{\prime \prime}=\inf _{\lambda, i: v_{i}^{\lambda}=1} c_{i}^{\lambda}
$$

в противном случае положим $b^{\prime \prime}=\infty$. Рассмотрим множества $V_{\lambda}^{\prime}=V_{\lambda} \backslash\{0,1\}$. Если $V_{\lambda}^{\prime} \neq \varnothing$ для некоторого индекса $\lambda$, то положим

$$
b=\inf _{\lambda, i: v_{i}^{\lambda} \in V_{\lambda}^{\prime}} c_{i}^{\lambda},
$$

в противном случае положим $b=\infty$. Положим

$$
\varepsilon_{F}^{\prime}=\min \left\{b^{\prime}, b\right\}, \quad \varepsilon_{F}^{\prime \prime}=\min \left\{b, b^{\prime \prime}\right\} .
$$

Скажем, что базовое множество предикатов $F$ и основание $\phi=\left\langle\mu, \mu^{\prime}\right\rangle$ согласованы, если

(1) для любых $A_{1}, \ldots, A_{m} \in\left\{N_{f}: f \in F\right\}$ таких, что

$$
\bigcap_{i=1}^{m} A_{i} \neq \varnothing
$$

справедливо соотношение

$$
\min \left\{\mu^{\prime}\left(A_{1}\right), \ldots, \mu^{\prime}\left(A_{m}\right)\right\} \equiv \mu^{\prime}\left(\bigcap_{i=1}^{m} A_{i}\right),
$$


(2) $\mu(x, 0) \equiv \mu^{\prime}(x)$ для всех $x \in\left(-b_{F}^{\prime}, b_{F}^{\prime \prime}\right)$, причем если $u_{F}^{\prime}=1$, то

$$
\mu\left(-b_{F}^{\prime}, 0\right)=\mu^{\prime}\left(-b_{F}^{\prime}\right),
$$

если $u_{F}^{\prime \prime}=1$, то

$$
\mu\left(b_{F}^{\prime \prime}, 0\right)=\mu^{\prime}\left(b_{F}^{\prime \prime}\right),
$$

если $u_{F}^{\prime}=u_{F}^{\prime \prime}=0$ и $u_{F}=1$, то

$$
\max \left\{\mu\left(-b_{F}^{\prime}, 0\right), \mu\left(b_{F}^{\prime \prime}, 0\right)\right\}=\max \left\{\mu^{\prime}\left(-b_{F}^{\prime}\right), \mu^{\prime}\left(b_{F}^{\prime \prime}\right)\right\},
$$

(3) для всех $y \neq 0$ и всех $x \in[-1,0]$ справедливо неравенство $\mu(x, y) \leqslant \varepsilon_{F}^{\prime}$, для всех $y \neq 0$ при всех $x \in[0,1]$ справедливо неравенство $\mu(x, y) \leqslant \varepsilon_{F}^{\prime \prime}$.

Назовем ИГ неизбыточным, если в нем нет ориентированных цепей, проводимость которых тождественно равна нулю.

Множество неизбыточных ИГ над базовым множеством $F$, допустимых для ЗИП $I$, обозначим $\mathcal{U}^{\prime}(I, \mathscr{F})$.

Скажем, что базовое множество предикатов $F$ и основание $\phi=\left\langle\mu, \mu^{\prime}\right\rangle$ вырождены, если для любого отношения $\rho \subseteq[0,1]^{2}$ и любой ЗИП $I$ типа $\langle[0,1],[0,1], \rho\rangle$ выполняется соотношение

$$
\left(U \in U^{\prime}(I, F) \Longrightarrow \phi(U) \in U(\phi(I), \phi(F))\right) .
$$

Теорема 3. Базовое множество предикатов $F$ и основание $\phi=\left\langle\mu, \mu^{\prime}\right\rangle$ вырождены тогда и только тогда, когда они согласованы.

\section{3. Критерий допустимости нечетких информационных графов}

В данном разделе приводится доказательство теоремы 1.

Начнем с доказательства необходимости. Пусть НИГ $U$ допустим для ЗНІП $I=\langle X, V, \eta\rangle$. Тогда по определению для любого запроса $x \in X$ и любого числа $c \in(0,1]$

$$
\mathscr{S}_{U}(x, c)=\{y \in V: \eta(x, y) \geqslant c\} .
$$

Пусть запись $y \in V$ такова, что $O(y, \eta) \neq \varnothing$. Это означает, что существуют $x \in O(y, \eta)$ и $c^{*} \in(0,1]$ такие, что $\eta(x, y)=c^{*}$. НИГ $U$ допустим для ЗНП $I$, значит, среди его листьев есть лист, которому соответствует запись $y$. Поэтому $L_{U}(y) \neq \varnothing$.

Пусть для некоторого $x \in X$

$$
\eta(x, y)<\max _{\alpha \in L_{U}(y)} \varphi_{\alpha}(x)=c^{\prime} .
$$

Тогда $\eta(x, y)<c^{\prime}$, следовательно $y \notin \mathscr{J}_{U}\left(x, c^{\prime}\right)$ (из определения допустимого НИГ для ЗНП), а из равенства

$$
\max _{\alpha \in L_{U}(y)} \varphi_{\alpha}(x)=c^{\prime}
$$

следует, что $y \in \mathscr{I}_{U}\left(x, c^{\prime}\right)$ (из определения $\mathscr{F}_{U}(x, c)$ ). Получаем противоречие.

Пусть теперь для некоторого $x \in X$

$$
\max _{\alpha \in L_{U}(y)} \varphi_{\alpha}(x)<\eta(x, y)=c^{\prime \prime} .
$$


Тогда

$$
\max _{\alpha \in L_{U}(y)} \varphi_{\alpha}(x)<c^{\prime \prime},
$$

следовательно, $y \notin \mathscr{F}_{U}\left(x, c^{\prime \prime}\right)$ (из определения $\mathscr{I}_{U}(x, c)$ ), а из равенства $\eta(x, y)=c^{\prime \prime}$ следует, что $y \in \mathscr{S}_{U}\left(x, c^{\prime \prime}\right)$ (из определения допустимого НИГ для ЗНП). Получаем противоречие.

Итак,

$$
\max _{\alpha \in L_{U}(y)} \varphi_{\alpha}(x) \equiv \eta(x, y) .
$$

Если же запись $y \in Y$ такова, что $O(y, \eta)=\varnothing$, то $\eta(x, y)=0$ для любого запроса $x \in X$. Поэтому для любого числа $c \in(0,1]$

$$
y \notin \mathscr{\mathscr { S }}(x, c)
$$

для любого запроса $x \in X$ (из определения допустимого НИГ для ЗНП), и либо $L_{U}(y)=$ $\varnothing$, либо

$$
\max _{\alpha \in L_{U}(y)} \varphi_{\alpha}(x) \equiv 0 .
$$

Необходимость доказана.

Докажем достаточность. Пусть выполняются услювия (1) и (2) теоремы.

Пусть для некоторого запроса $x \in X$, некоторой записи $y \in V$ и некоторого числа $c \in(0,1]$

$$
\max _{\alpha \in L_{U}(y)} \varphi_{\alpha}(x) \equiv \eta(x, y) \geqslant c .
$$

Тогда $y \in \mathscr{S}_{U}(x, c)$ (по определению $\mathscr{S}_{U}(x, c)$ ) и одновременно

$$
y \in\{y \in V: \eta(x, y) \geqslant c\},
$$

если же

$$
\max _{\alpha \in L_{U}(y)} \varphi_{\alpha}(x) \equiv \eta(x, y)<c,
$$

то $y \notin\{y \in V: \eta(x, y) \geqslant c\}$. Поэтому для любого запроса $x \in X$ и для любого числа $c \in(0,1]$

$$
\mathscr{I}_{U}(x, c)=\{y \in V: \eta(x, y) \geqslant c\},
$$

то есть НИГ $U$ допустим для ЗНП $I=\langle X, V, \eta\rangle$.

Теорема доказана.

Отметим, что понятие ЗИП, введенное выше, является частным случаем понятия ЗНП. Действительно, ЗИП $I=\langle X, Y, \rho\rangle$ можно рассматривать как ЗНП $I=\langle X, Y, \rho(x, y)\rangle$ с отношением нечеткого поиска

$$
\rho(x, y)= \begin{cases}1, & x \rho y \\ 0 & \text { в противном случае }\end{cases}
$$

Базовое множество предикатов $F$ можно рассматривать как базовое множество нечетких функщий $F$, а любой ИГ $U$ над $F$ как НИГ $U$ над $F$. В таком случае для любого $x \in X$ для любого $c \in(0,1]$ выполняется равенство

$$
\{y \in V: \rho(x, y) \geqslant c\}=\{y \in V: x \rho y\}
$$


следовательно, для ЗИП $I=\langle X, Y, \rho\rangle$

$$
\mathscr{F}_{U}(x) \equiv \mathscr{I}_{U}(x, c)
$$

для любого $c \in(0,1]$. Поэтому ИГ $U$ допустим для ЗИП $I=\langle X, Y, \rho\rangle$ тогда и только тогда, когда НИГ $U$ допустим для ЗНП $I=\langle X, Y, \rho(x, y)\rangle$. Поэтому теорема 1 справедлива для ИГ и дальше будет использоваться для ИГ без комментариев.

\section{4. Критерий полноты базового множества}

В данном разделе приводится доказательство теоремы 2.

Начнем с доказательства необходимости. Пусть базовое множество F्F полно для типа $S=\langle X, Y, \eta\rangle$. Возьмем произвольную запись $y \in Y$ такую, что $O(y, \eta) \neq \varnothing$. Пусть $V=\{y\}$. Рассмотрим ЗНП $I=\langle X, V, \eta\rangle$. Существует НИГ $U$ над базовым множеством F, допустимый для ЗНП I. Рассмотрим $L_{U}(y)$. По теореме 1 это множество не пусто и

$$
\max _{\alpha \in L_{U}(y)} \varphi_{\alpha}(x) \equiv \eta(x, y) \text {. }
$$

Но каждая $\varphi_{\alpha}(x)$ по определению равна максимуму проводимостей ориентированных цепей, ведущих из корня в вершину $\alpha$. Занумеруем все ориентированные цепи, ведущие из корня в вершины из $L_{U}(y)$ (в силу того, что $U$ есть конечный граф, таких цепей конечное число). В каждой цепи занумеруем ребра (их тоже конечное число). Функцию, приписанную $j$-му ребру в $i$-й цепи, обозначим $f_{i j}$. Тогда

$$
\begin{aligned}
\eta(x, y) \equiv \max _{\alpha \in L_{U}(y)} \varphi_{\alpha}(x) & =\max _{\alpha \in L_{U}(y)} \max _{i \in I_{\alpha}} \min _{1 \leqslant j \leqslant m_{i}} f_{i j}(x) \\
& =\max _{1 \leqslant i \leqslant n} \min _{1 \leqslant j \leqslant m_{i}} f_{i j}(x),
\end{aligned}
$$

где $I_{\alpha}$ есть множество номеров, соответствующих ориентированным цепям, ведущим из корня в вершину $\alpha, m_{i}$ есть число ребер в $i$-й цепи, $n$ есть число ориентированных цепей из корня в вершины из $L_{U}(y)$.

Необходимость доказана.

Докажем теперь достаточность. Возьмем произвольную ЗНП $I=\langle X, V, \eta\rangle$, где $V=$ $\left\{y_{1}, y_{2}, \ldots, y_{k}\right\}$. Построим НИГ $U$, допустимый для ЗНП $I$.

Для любой записи $y_{l} \in V$ такой, что $O\left(y_{l}, \eta\right) \neq \varnothing$, функция $\eta\left(x, y_{l}\right)$ как функция от $x$ может быть представлена формулой вида

$$
\eta\left(x, y_{l}\right)=\max _{1 \leqslant i \leqslant n_{l}} \min _{1 \leqslant j \leqslant m_{l i}} f_{i j}^{l}(x),
$$

где $f_{i j}^{l} \in \mathscr{F}$.

Возьмем $k+1$ вершину, выделим корень, остальные $k$ вершин назовем листьями и занумеруем числами $1,2, \ldots, k$.

Для всех $l$ от 1 до $k$ припишем листу с номером $l$ (обозначим его $\alpha_{l}$ ) запись $y_{l}$. Если $O\left(y_{l}, \eta\right) \neq \varnothing$, то проведем из корня в лист $\alpha_{l}$ ровно $n_{l}$ цепей, причем $i$-я цепь состоит из $m_{l i}$ ребер. Припишем $j$-му ребру в $i$-й цепи функцию $f_{i j}^{l}$. НИГ $U$ построен.

Тогда функция фильтра $l$-й вершины имеет вид

$$
\varphi_{\alpha_{l}}(x)=\max _{1 \leqslant i \leqslant n_{l}} \min _{1 \leqslant j \leqslant m_{l i}} f_{i j}^{l}(x)
$$


то есть $\varphi_{\alpha_{l}}(x)=\eta\left(x, y_{l}\right)$. С другой стороны, для любой вершины $y \in V$ такой, что $O(y, \eta)=\varnothing$, множество $L_{U}(y)$ пусто. Таким образом, по теореме 1 НИГ $U$ допустим для उНП $I$.

Произвольность выбора ЗНП I доказывает достаточность.

Теорема доказана.

\section{5. Критерий вырожденности основания перехода}

Замыкание произвольного множества $A$ вещественной прямой будем обозначать [ $A]$.

Скажем, что $x_{0}$ является точкой слабого максимума функции $f$, если выполняется одно из следующих условий:

- $x_{0}$ является точкой локального максимума функции $f$,

- $f$ возрастает в левой полуокрестности точки $x_{0}$ и убывает в правой полуокрестности точки $x_{0}$,

- $f$ возрастает в левой полуокрестности и в правой полуокрестности точки $x_{0}$, но $f\left(x_{0}-0\right)>f\left(x_{0}+0\right)$,

- $f$ убывает в левой и в правой полуокрестности точки $x_{0}$, но $f\left(x_{0}-0\right)<f\left(x_{0}+0\right)$.

Лемма 1. Для произвольного множества $A \subseteq[0,1]$ все слабые максимумы функции $\mu^{\prime}(A)(x)$ принадлежат $[A]$.

Доказательство. Множество [A] можно представить в виде объединения не более чем счетного числа непересекающихся отрезков (см. [3], с. 60). Рассмотрим такое представление

$$
[A]=\bigcup_{i}\left[a_{i}^{1}, a_{i}^{2}\right]
$$

где $a_{i}^{1} \leqslant a_{i}^{2}$. Тогда

$$
\begin{aligned}
\mu^{\prime}(A)(x) & =\sup _{x^{\prime} \in A} \mu^{\prime}\left(x-x^{\prime}\right)=\sup _{x^{\prime} \in[A]} \mu^{\prime}\left(x-x^{\prime}\right)=\sup _{x^{\prime} \in \bigcup_{i}\left[a_{i}^{1}, a_{i}^{2}\right]} \mu^{\prime}\left(x-x^{\prime}\right) \\
& =\sup _{i} \sup _{x^{\prime} \in\left[a_{i}^{1}, a_{i}^{2}\right]} \mu^{\prime}\left(x-x^{\prime}\right)=\sup _{i} \mu^{\prime}\left(\left[a_{i}^{1}, a_{i}^{2}\right]\right)(x) .
\end{aligned}
$$

Индукцией по числу $n$ компонент связности в множестве [A] покажем, что $\mu^{\prime}(A) \equiv \mu^{\prime}([A])$ не имеет слабых максимумов вне $[A]$. При $n=1$ справедливо равенство $[A]=\left[a_{1}^{1}, a_{1}^{2}\right]$, на $\left[0, a_{1}^{1}\right]$

$$
\mu^{\prime}(A)(x) \equiv \sup _{x^{\prime} \in A} \mu^{\prime}\left(x-x^{\prime}\right)=\mu^{\prime}\left(x-a_{1}^{1}\right)
$$

- монотонно возрастающая функция, на $\left[a_{1}^{2}, 1\right]$

$$
\mu^{\prime}(A)(x) \equiv \mu^{\prime}\left(x-a_{1}^{2}\right)
$$


- монотонно убывающая функщия, и $\mu^{\prime}(A)(x) \equiv 1$ на $\left[a_{1}^{1}, a_{1}^{2}\right]$. Следовательно, все слабые максимумы функщии $\mu^{\prime}(A)$ принадлежат [A]. Предположим, что утверждение леммы доказано для не более, чем $n$ компонент связности $[A]$. Рассмотрим

$$
[A]=\bigcup_{i=1}^{n+1}\left[a_{i}^{1}, a_{i}^{2}\right]
$$

где $a_{i}^{1} \leqslant a_{i}^{2}<a_{i+1}^{1}$. Если $\mu^{\prime}(x) \equiv 0$ при $x \in[-1,0) \cup(0,1]$, то

$$
\mu^{\prime}([A])(x)= \begin{cases}1, & x \in[A], \\ 0, & x \in[0,1] \backslash[A],\end{cases}
$$

и, следовательно, утверждение леммы справедливо. Если $\mu^{\prime}(x)$ не есть тождественный ноль при $x \in[-1,0) \cup(0,1]$, то положим $x_{0}=\inf x$, где нижняя грань берется по всем $x \in\left[0, a_{n+1}^{1}\right]$ таким, что

$$
\mu^{\prime}\left(\left[a_{n+1}^{1}, a_{n+1}^{2}\right]\right)(x)>\mu^{\prime}\left(\bigcup_{i=1}^{n}\left[a_{i}^{1}, a_{i}^{2}\right]\right)(x)
$$

$\mathrm{Ha}\left[0, x_{0}\right)$

$$
\mu^{\prime}\left(\left[a_{n+1}^{1}, a_{n+1}^{2}\right]\right)(x) \leqslant \mu^{\prime}\left(\bigcup_{i=1}^{n}\left[a_{i}^{1}, a_{i}^{2}\right]\right)(x)
$$

на $\left(x_{0}, 1\right]$

$$
\mu^{\prime}\left(\left[a_{n+1}^{1}, a_{n+1}^{2}\right]\right)(x) \geqslant \mu^{\prime}\left(\bigcup_{i=1}^{n}\left[a_{i}^{1}, a_{i}^{2}\right]\right)(x) .
$$

Следовательно, на $\left[0, x_{0}\right)$

$$
\mu^{\prime}([A])(x) \equiv \mu^{\prime}\left(\bigcup_{i=1}^{n}\left[a_{i}^{1}, a_{i}^{2}\right]\right)(x),
$$

а на $\left(x_{0}, 1\right]$

$$
\mu^{\prime}([A])(x) \equiv \mu^{\prime}\left(\left[a_{n+1}^{1}, a_{n+1}^{2}\right]\right)(x) .
$$

По индуктивному предположению

$$
\mu^{\prime}\left(\bigcup_{i=1}^{n}\left[a_{i}^{1}, a_{i}^{2}\right]\right)(x)
$$

не имеет слабых максимумов вне

$$
\bigcup_{i=1}^{n}\left[a_{i}^{1}, a_{i}^{2}\right]
$$

a

$$
\mu^{\prime}\left(\bigcup_{i=1}^{n+1}\left[a_{i}^{1}, a_{i}^{2}\right]\right)(x)
$$


не имеет слабых максимумов вне $\left[a_{n+1}^{1}, a_{n+1}^{2}\right] . \mathrm{Ha}\left[a_{n}^{2}, 1\right]$

$$
\mu^{\prime}\left(\bigcup_{i=1}^{n}\left[a_{i}^{1}, a_{i}^{2}\right]\right)(x)=\mu^{\prime}\left(x-a_{n}^{2}\right)
$$

- монотонно убывающая функция, на $\left[0, a_{n+1}^{1}\right]$

$$
\mu^{\prime}\left(\left[a_{n+1}^{1}, a_{n+1}^{2}\right]\right)(x)=\mu^{\prime}\left(x-a_{n+1}^{1}\right)
$$

- монотонно возрастающая функция. Поэтому $x_{0}$ - точка локального минимума $\mu^{\prime}([A])(x)$. Следовательно, все слабые максимумы функции $\mu^{\prime}([A])(x)$ принадлежат $[A]$. Переходя к пределу по $n$, получаем утверждение леммы.

Следствие 1. Пусть $A \subseteq[0,1], c \in[0,1]$, и пусть

$$
(x, y) \in \rho \Longleftrightarrow(x \in A) \&(y=c) \text {. }
$$

Тогда все слабые максимумы функиии $\phi(\rho)(x, c)$ принадлежат $[A]$.

Доказательство. Рассмотрим

$$
\phi(\rho)(x, c)=\sup _{\left(x_{0}, y_{0}\right) \in \rho} \mu\left(x-x_{0}, c-y_{0}\right)=\sup _{x_{0} \in A} \mu\left(x-x_{0}, 0\right) .
$$

Введем функцию $\tilde{\mu}(x)=\mu(x, 0)$. Заметим, что $\tilde{\mu}$ обладает теми же свойствами, что и функция $\mu^{\prime}$, а именно,

$$
\tilde{\mu}(x)=1 \Longleftrightarrow x=0
$$

и для любого $x \in[-1,1]$ и любого $\lambda \in(0,1)$ выполняется неравенство $\tilde{\mu}(\lambda x)>\tilde{\mu}(x)$. Для произвольного множества $B \subseteq[0,1]$ положим

$$
\tilde{\mu}(B)=\sup _{x_{0} \in B} \tilde{\mu}\left(x-x_{0}\right) \text {. }
$$

Тогда $\phi(\rho)(x, c)=\tilde{\mu}(A)(x)$. Теперь достаточно показать, что все слабые максимумы функции $\tilde{\mu}(A)(x)$ принадлежат [A]. Доказательство этого факта совпадает с доказательством леммы 1 , только вместо функции $\mu^{\prime}$ рассматривается функция $\tilde{\mu}$.

Следствие 2. Пусть $A-$ непустое подмножество [0,1]. В точках слабого максимума функиия $\mu^{\prime}(A)$ принимает значение 1.

Лемма 2. Если $f_{1}, \ldots, f_{m}-$ предикаты, то либо

$$
\phi\left(f_{1} \& \ldots \& f_{m}\right) \equiv \min _{1 \leqslant i \leqslant m} \phi\left(f_{i}\right),
$$

либо $\min _{1 \leqslant i \leqslant m} \phi\left(f_{i}\right)$ имеет слабый максимум, не принадлежащий $\left[\bigcap_{i=1}^{m} N_{f_{i}}\right]$.

Доказательство. Для любых предикатов $f_{1}, \ldots, f_{m}$ справедливо неравенство

$$
\phi\left(f_{1} \& \ldots \& f_{m}\right) \leqslant \min _{1 \leqslant i \leqslant m} \phi\left(f_{i}\right)
$$

Пусть

$$
\phi\left(f_{1} \& \ldots \& f_{m}\right) \equiv \min _{1 \leqslant i \leqslant m} \phi\left(f_{i}\right)
$$


Тогда в силу леммы 1 все слабые максимумы функции $\phi\left(f_{1} \& \ldots \& f_{m}\right)$, а следовательно, и функции $\min _{1 \leqslant i \leqslant m} \phi\left(f_{i}\right)$, принадлежат $\left[\bigcap_{i=1}^{m} N_{f_{i}}\right]$.

Предположим, что существует точка $x_{0}$ такая, что

$$
\phi\left(f_{1} \& \ldots \& f_{m}\right)\left(x_{0}\right)<\min _{1 \leqslant i \leqslant m} \phi\left(f_{i}\right)\left(x_{0}\right) .
$$

Индукщией по числу $m$ предикатов покажем, что в таком случае $\min _{1 \leqslant i \leqslant m} \phi\left(f_{i}\right)$ имеет слабый максимум, не принадлежащий $\left[\bigcap_{i=1}^{m} N_{f_{i}}\right]$.

При $m=2$ возможны следующие случаи.

Случай 1. Пусть $\min \left\{\phi\left(f_{1}\right)\left(x_{0}\right), \phi\left(f_{2}\right)\left(x_{0}\right)\right\}=1$. Следовательно, в силу того, что $\min \left\{\phi\left(f_{1}\right)(x), \phi\left(f_{2}\right)(x)\right\} \leqslant 1$ для всех $x$, точка $x_{0}$ есть точка слабого максимума функции $\min \left\{\phi\left(f_{1}\right), \phi\left(f_{2}\right)\right\}$. С другой стороны,

$$
\phi\left(f_{1} \& f_{2}\right)\left(x_{0}\right)<\min \left\{\phi\left(f_{1}\right)\left(x_{0}\right), \phi\left(f_{2}\right)\left(x_{0}\right)\right\}=1,
$$

поэтому $x_{0} \notin\left[N_{f_{1}} \cap N_{f_{2}}\right]$, так как $\phi\left(f_{1} \& f_{2}\right)(x)=1$ для всех $x \in\left[N_{f_{1}} \cap N_{f_{2}}\right]$.

Случай 2. Пусть $\min \left\{\phi\left(f_{1}\right)\left(x_{0}\right), \phi\left(f_{2}\right)\left(x_{0}\right)\right\}<1$. Без ограничения общности можно считать, что

$$
\min \left\{\phi\left(f_{1}\right)\left(x_{0}\right), \phi\left(f_{2}\right)\left(x_{0}\right)\right\}=\phi\left(f_{1}\right)\left(x_{0}\right) .
$$

В силу следствия 2 леммы $1 x_{0}$ не может быть точкой слабого максимума функции $\phi\left(f_{1}\right)$. Пусть $\phi\left(f_{1}\right)$ возрастает в точке $x_{0}$ (случай, когда $\phi\left(f_{1}\right)$ убывает в точке $x_{0}$, рассматривается аналогично). В силу леммы 1 существует точка $x_{1}>x_{0}$ такая, что $\phi\left(f_{1}\right)$ монотонно возрастает на $\left[x_{0}, x_{1}\right]$ и $\phi\left(f_{1}\right)\left(x_{1}\right)=1$. Понятно, что на $\left[x_{0}, x_{1}\right)$ нет точек из $\left[N_{f_{1}}\right]$. Поэтому на $\left[x_{0}, x_{1}\right]$ выполняется соотношение

$$
\phi\left(f_{1}\right)(x) \equiv \sup _{x \in N_{f_{1}}} \mu^{\prime}\left(x-x^{\prime}\right)=\mu^{\prime}\left(x-x_{1}\right)
$$

Пусть $\phi\left(f_{2}\right)\left(x_{1}\right)=1$, тогда и $\min \left\{\phi\left(f_{1}\right)\left(x_{1}\right), \phi\left(f_{2}\right)\left(x_{1}\right)\right\}=1$. Докажем, что при этом $\phi\left(f_{1} \& f_{2}\right)\left(x_{1}\right)<1$. Предположим, что $\phi\left(f_{1} \& f_{2}\right)\left(x_{1}\right)=1$. Тогда $x_{1} \in\left[N_{f_{1}} \cap N_{f_{2}}\right]$, и

$$
\phi\left(f_{1} \& f_{2}\right)\left(x_{0}\right)=\sup _{x^{\prime} \in N_{f_{1}} \cap N_{f_{2}}} \mu^{\prime}\left(x_{0}-x^{\prime}\right) \geqslant \mu^{\prime}\left(x_{0}-x_{1}\right)=\phi\left(f_{1}\right)\left(x_{0}\right),
$$

следовательно, $\phi\left(f_{1} \& f_{2}\right)\left(x_{0}\right)=\min \left\{\phi\left(f_{1}\right)\left(x_{0}\right), \phi\left(f_{2}\right)\left(x_{0}\right)\right\}$, и мы получаем противоречие. Значит,

$$
\phi\left(f_{1} \& f_{2}\right)\left(x_{1}\right)<\min \left\{\phi\left(f_{1}\right)\left(x_{1}\right), \phi\left(f_{2}\right)\left(x_{1}\right)\right\}=1 .
$$

Следовательно, этот случай сводится к случаю 1 для точки $x_{1}$.

Пусть $\phi\left(f_{2}\right)\left(x_{1}\right)<1$ и $\phi\left(f_{1}\right)\left(x_{0}\right)=\phi\left(f_{2}\right)\left(x_{0}\right)$. Тогда, так как $x_{1} \notin\left[N_{f_{2}}\right]$, существует точка $x_{2} \in\left[0, x_{0}\right)$ такая, что $\phi\left(f_{2}\right)\left(x_{2}\right)=1$ и $\phi\left(f_{2}\right)(x) \equiv \mu^{\prime}\left(x-x_{2}\right)$ на $\left[x_{2}, x_{0}\right]$. Покажем, что $x_{2} \notin\left[N_{f_{1}}\right]$. Действительно, если $x_{2} \in\left[N_{f_{1}}\right]$, то

$$
\phi\left(f_{1} \& f_{2}\right)\left(x_{0}\right) \geqslant \mu^{\prime}\left(x_{0}-x_{2}\right)=\phi\left(f_{2}\right)\left(x_{0}\right)=\phi\left(f_{1}\right)\left(x_{0}\right)=\min \left\{\phi\left(f_{1}\right)\left(x_{0}\right), \phi\left(f_{2}\right)\left(x_{0}\right)\right\},
$$

следовательно,

$$
\phi\left(f_{1} \& f_{2}\right)\left(x_{0}\right)=\min \left\{\phi\left(f_{1}\right)\left(x_{0}\right), \phi\left(f_{2}\right)\left(x_{0}\right)\right\},
$$

и мы опять получаем противоречие. 
Понятно, что $\left[x_{2}, x_{1}\right) \cap\left[N_{f_{1}}\right]=\varnothing$ и $\left(x_{2}, x_{1}\right] \cap\left[N_{f_{2}}\right]=\varnothing$. Следовательно,

$$
\phi\left(f_{1}\right)\left(x_{0}-0\right)<\phi\left(f_{1}\right)\left(x_{0}\right), \quad \phi\left(f_{2}\right)\left(x_{0}+0\right)<\phi\left(f_{2}\right)\left(x_{0}\right),
$$

так что $x_{0}$ - точка слабого максимума функции $\min \left\{\phi\left(f_{1}\right)(x), \phi\left(f_{2}\right)(x)\right\}$ и, очевидно, $x_{0} \notin\left[N_{f_{1}} \cap N_{f_{2}}\right]$.

Пусть $\phi\left(f_{2}\right)\left(x_{1}\right)<1$ и $\phi\left(f_{1}\right)\left(x_{0}\right)<\phi\left(f_{2}\right)\left(x_{0}\right)$. Существует точка $x_{2} \in\left[0, x_{1}\right)$ такая, что $\phi\left(f_{2}\right)\left(x_{2}\right)=1$ и $\phi\left(f_{2}\right)(x) \equiv \mu^{\prime}\left(x-x_{2}\right)$ на $\left[x_{0}, x_{2}\right]\left(\left[x_{2}, x_{0}\right]\right)$. Понятно, что $\left[x_{2}, x_{1}\right) \cap N_{f_{1}}=\varnothing$. Пусть $x_{3}=\sup _{x \in N_{f_{2}} \cap\left(x_{2}, x_{1}\right)} x$. Так как $\phi\left(f_{2}\right)\left(x_{1}\right)<1$, то $x_{3} \neq x_{1}$. Тогда $\phi\left(f_{2}\right)(x) \equiv \mu^{\prime}\left(x-x_{3}\right)-$ монотонно убывающая функщия на $\left[x_{3}, x_{1}\right]$. На $\left[x_{0}, x_{1}\right]$ функщия $\phi\left(f_{1}\right)(x) \equiv \mu^{\prime}\left(x-x_{1}\right)$ монотонно возрастает. Так как $\phi\left(f_{1}\right)\left(x_{0}\right)<\phi\left(f_{2}\right)\left(x_{0}\right)$, а $\phi\left(f_{2}\right)\left(x_{1}\right)<\phi\left(f_{1}\right)\left(x_{1}\right)=1$, существует точка $x^{*} \in\left(x_{0}, x_{1}\right) \cap\left(x_{3}, x_{1}\right)$ такая, что на отрезке $\left[x_{0}, x^{*}\right]$ функция $\min \left\{\phi\left(f_{1}\right)(x), \phi\left(f_{2}\right)(x)\right\}=\phi\left(f_{1}\right)(x)$ монотонно возрастает, а на отрезке $\left[x^{*}, x_{1}\right]$ функция $\min \left\{\phi\left(f_{1}\right)(x), \phi\left(f_{2}\right)(x)\right\}=\phi\left(f_{2}\right)(x)$ монотонно убывает. Тогда $x^{*}$ - точка слабого максимума функции $\min \left\{\phi\left(f_{1}\right)(x), \phi\left(f_{2}\right)(x)\right\}$, и, очевидно, $x^{*} \notin\left[N_{f_{1}} \cap N_{f_{2}}\right]$.

Если $\phi\left(f_{1}\right)$ имеет в точке $x_{0}$ локальный минимум, то в силу леммы 1 существуют точки $x^{\prime}$ и $x^{\prime \prime}$ такие, что $\phi\left(f_{1}\right)\left(x^{\prime}\right)=\phi\left(f_{1}\right)\left(x^{\prime \prime}\right)=1, \phi\left(f_{1}\right)$ убывает на интервале $\left(x^{\prime}, x_{0}\right)$ и возрастает на интервале $\left(x_{0}, x^{\prime \prime}\right)$. Понятно, что $\phi\left(f_{1}\right)(x) \equiv \mu^{\prime}\left(x-x^{\prime}\right)$ на $\left[x^{\prime}, x_{0}\right)$, а на $\left(x_{0}, x^{\prime \prime}\right]$ справедливы соотношения

$$
\begin{aligned}
\phi\left(f_{1}\right)(x) & \equiv \mu^{\prime}\left(x-x^{\prime \prime}\right), \\
\phi\left(f_{1}\right)\left(x_{0}\right) & =\max \left\{\mu^{\prime}\left(x_{0}-x^{\prime}\right), \mu^{\prime}\left(x_{0}-x^{\prime \prime}\right)\right\} .
\end{aligned}
$$

Если $\phi\left(f_{1}\right)\left(x_{0}\right)=\mu^{\prime}\left(x_{0}-x^{\prime}\right)$, то положим $x_{1}=x^{\prime}$, в противном случае положим $x_{1}=x^{\prime \prime}$. Дальше доказательство совпадает с описанным выше.

Базис индукции доказан.

Проведем индуктивный переход. Пусть лемма справедлива для не более, чем $m$ предикатов. Тогда либо

$$
\phi\left(f_{1} \& \ldots \& f_{m}\right) \equiv \min _{1 \leqslant i \leqslant m} \phi\left(f_{i}\right),
$$

либо $\min _{1 \leqslant i \leqslant m} \phi\left(f_{i}\right)$ имеет слабый максимум, не принадлежащий $\left[\bigcap_{i=1}^{m} N_{f_{i}}\right]$.

Если для всех $x \in[0,1]$

$$
\min _{1 \leqslant i \leqslant m} \phi\left(f_{i}\right) \leqslant \phi\left(f_{m+1}\right)
$$

To

$$
\min _{1 \leqslant i \leqslant m+1} \phi\left(f_{i}\right) \equiv \min _{1 \leqslant i \leqslant m} \phi\left(f_{i}\right) .
$$

Понятно, что в таком случае $\left[\bigcap_{i=1}^{m+1} N_{f_{i}}\right]=\left[\bigcap_{i=1}^{m} N_{f_{i}}\right]$, слабые максимумы функций $\min _{1 \leqslant i \leqslant m+1} \phi\left(f_{i}\right)(x)$ и $\min _{1 \leqslant i \leqslant m} \phi\left(f_{i}\right)(x)$ совпадают, и по предположению индукции утверждение леммы верно.

Если для всех $x \in[0,1]$

$$
\min _{1 \leqslant i \leqslant m} \phi\left(f_{i}\right) \geqslant \phi\left(f_{m+1}\right)
$$

то $\min _{1 \leqslant i \leqslant m+1} \phi\left(f_{i}\right) \equiv \phi\left(f_{m+1}\right)$. Понятно, что в таком случае $\left[\bigcap_{i=1}^{m+1} N_{f_{i}}\right]=\left[N_{f_{m+1}}\right]$, слабые максимумы функции $\min _{1 \leqslant i \leqslant m+1} \phi\left(f_{i}\right)(x)$ совпадают со слабыми максимумами функции $\phi\left(f_{m+1}\right)(x)$, и по предположению индукции утверждение леммы верно. 
Пусть теперь $\min _{1 \leqslant i \leqslant m} \phi\left(f_{i}\right)(x)-\phi\left(f_{m+1}\right)(x)$ меняет знак на $[0,1]$. Положим

$$
\begin{gathered}
X_{0}=\left\{x \in[0,1]:\left(\min _{1 \leqslant i \leqslant m} \phi\left(f_{i}\right)(x+0)<\phi\left(f_{m+1}\right)(x+0)\right)\right. \\
\left.\&\left(\min _{1 \leqslant i \leqslant m} \phi\left(f_{i}\right)(x-0)>\phi\left(f_{m+1}\right)(x-0)\right)\right\}, \\
X_{1}=\left\{x \in[0,1]:\left(\min _{1 \leqslant i \leqslant m} \phi\left(f_{i}\right)(x+0)>\phi\left(f_{m+1}\right)(x+0)\right)\right. \\
\left.\&\left(\min _{1 \leqslant i \leqslant m} \phi\left(f_{i}\right)(x-0)<\phi\left(f_{m+1}\right)(x-0)\right)\right\} .
\end{gathered}
$$

Понятно, что $X_{0} \cup X_{1} \neq \varnothing$. Без ограничения общности можно считать, что $X_{0} \neq \varnothing$. Возьмем произвольную точку $x_{0} \in X_{0}$. Предположим, что $\phi\left(f_{m+1}\right)$ убывает в $x_{0}$, тогда $\min _{1 \leqslant i \leqslant m} \phi\left(f_{i}\right)$ тоже убывает в $x_{0}$. Согласно лемме 1 существует точка $x_{1}$ такая, что $\phi\left(f_{m+1}\right)(x) \equiv \mu^{\prime}\left(x-x_{1}\right)$ на $\left[x_{1}, x_{0}+\delta_{1}\right], \delta_{1}>0$. Так как

$$
\min _{1 \leqslant i \leqslant m} \phi\left(f_{i}\right)\left(x_{0}-0\right)>\phi\left(f_{m+1}\right)\left(x_{0}-0\right)
$$

и $\min _{1 \leqslant i \leqslant m} \phi\left(f_{i}\right)$ убывает в $x_{0}$, должна существовать точка $x_{2} \in\left(x_{1}, x_{0}\right]$ такая, что для некоторых $\delta_{2}>0, \delta_{3}>0$

$$
\min _{1 \leqslant i \leqslant m} \phi\left(f_{i}\right)(x) \equiv \mu^{\prime}\left(x-x_{2}\right)
$$

на $\left[x_{0}-\delta_{2}, x_{0}+\delta_{3}\right]$ (так как значение функщии $\min _{1 \leqslant i \leqslant m} \phi\left(f_{i}\right)$ на интервале, где она монотонна, определяется значением на этом интервале по крайней мере одной из функций $\left.\phi\left(f_{i}\right), i=1, \ldots, m\right)$. Но тогда

$$
\phi\left(f_{m+1}\right)(x)<\min _{1 \leqslant i \leqslant m} \phi\left(f_{i}\right)(x)
$$

на $\left[x_{0}, x_{0}+\min \left\{\delta_{1}, \delta_{3}\right\}\right]$, получаем противоречие.

Предположим, что $x_{0}$ - точка локального минимума $\phi\left(f_{m+1}\right)$. Согласно лемме 1 существуют точка $x_{1}$ такая, что

$$
\phi\left(f_{m+1}\right)(x) \equiv \mu^{\prime}\left(x-x_{1}\right)
$$

на $\left[x_{1}, x_{0}\right)$ и точка $x_{2} \in\left(x_{0}, 1\right]$ такая, что

$$
\phi\left(f_{m+1}\right)(x) \equiv \mu^{\prime}\left(x-x_{2}\right)
$$

на $\left(x_{0}, x_{2}\right]$. Так как

$$
\min _{1 \leqslant i \leqslant m} \phi\left(f_{i}\right)(x-0)>\phi\left(f_{m+1}\right)(x-0),
$$

должна существовать точка $x_{3} \in\left(x_{1}, x_{0}\right]$ такая, что для некоторого $\delta>0$

$$
\min _{1 \leqslant i \leqslant m} \phi\left(f_{i}\right)(x) \equiv \mu^{\prime}\left(x-x_{3}\right)
$$

на $\left[x_{0}-\delta, x_{0}\right)$. Для любого $x \in\left[x_{3}, x_{0}+\left(x_{3}-x_{1}\right)\right)$

$$
\phi\left(f_{m+1}\right)\left(x_{0}-0\right)=\mu^{\prime}\left(x_{0}-x_{1}-0\right) \leqslant \mu^{\prime}\left(x-x_{3}\right) .
$$

Кроме того,

$$
\phi\left(f_{m+1}\right)\left(x_{0}+0\right)=\mu^{\prime}\left(x_{0}-x_{2}+0\right)>\min _{1 \leqslant i \leqslant m} \phi\left(f_{i}\right)\left(x_{0}+0\right) \geqslant \mu^{\prime}\left(x_{0}-x_{3}+0\right) .
$$


Поэтому $\phi\left(f_{m+1}\right)\left(x_{0}+0\right)>\phi\left(f_{m+1}\right)\left(x_{0}-0\right)$. Пусть

$$
\phi\left(f_{m+1}\right)\left(x_{0}+0\right)-\phi\left(f_{m+1}\right)\left(x_{0}-0\right)=\alpha .
$$

Тогда если $x_{2}<1$, то для любого $x_{4} \in\left(x_{2}, 1\right]$

$$
\mu^{\prime}\left(x_{0}-x_{4}+0\right)<\mu^{\prime}\left(x_{0}-x_{2}+0\right)-\alpha .
$$

Но $\mu^{\prime}\left(x_{0}-x_{3}\right)>\mu^{\prime}\left(x_{0}-x_{2}-0\right)=\mu^{\prime}\left(x_{0}-x_{2}+0\right)-\alpha$. Поэтому $\min _{1 \leqslant i \leqslant m} \phi\left(f_{i}\right)$ убывает в правой полуокрестности $x_{0}$. При этом $\phi\left(f_{m+1}\right)$ убывает в левой полуокрестности $x_{0}$. Заметим, что

$$
\begin{aligned}
\phi\left(f_{m+1}\right)\left(x_{0}-0\right) & =\mu^{\prime}\left(x_{0}-x_{1}-0\right), \\
\min _{1 \leqslant i \leqslant m} \phi\left(f_{i}\right)\left(x_{0}+0\right) & =\mu^{\prime}\left(x_{0}-x_{3}+0\right) .
\end{aligned}
$$

Так как $x_{0}-x_{3}<x_{0}-x_{1}$, то $\mu^{\prime}\left(x_{0}-x_{3}+0\right)>\mu^{\prime}\left(x_{0}-x_{1}-0\right)$. Поэтому

$$
\begin{aligned}
\min _{1 \leqslant i \leqslant m+1} \phi\left(f_{i}\right)\left(x_{0}-0\right) & =\phi\left(f_{m+1}\right)\left(x_{0}-0\right) \\
& <\min _{1 \leqslant i \leqslant m} \phi\left(f_{i}\right)\left(x_{0}+0\right)=\min _{1 \leqslant i \leqslant m+1} \phi\left(f_{i}\right)\left(x_{0}+0\right) .
\end{aligned}
$$

Следовательно, $x_{0}$ является точкой слабого максимума функции $\min _{1 \leqslant i \leqslant m+1} \phi\left(f_{i}\right)$. При этом, так как $x_{0}$ не является точкой максимума $\phi\left(f_{m+1}\right)$,

$$
x_{0} \notin\left[\bigcap_{i=1}^{m+1} N_{f_{i}}\right] \text {. }
$$

Наконец, предположим, что $\phi\left(f_{m+1}\right)$ возрастает в $x_{0}$, тогда очевидно, что функция $\min _{1 \leqslant i \leqslant m} \phi\left(f_{i}\right)$ убывает в $x_{0}$. Следовательно, $x_{0}$ является точкой слабого максимума функции $\min _{1 \leqslant i \leqslant m+1} \phi\left(f_{i}\right)$. При этом, так как $x_{0}$ не является точкой максимума $\phi\left(f_{m+1}\right)$,

$$
x_{0} \notin\left[\bigcap_{i=1}^{m+1} N_{f_{i}}\right] \text {. }
$$

Аналогично, если $X_{1} \neq \varnothing$ и $x_{1} \in X_{1}$, то $x_{1}$ является точкой слабого максимума функции $\min _{1 \leqslant i \leqslant m+1} \phi\left(f_{i}\right)$, но, так как $x_{1}$ не является точкой максимума $\phi\left(f_{m+1}\right)$,

$$
x_{1} \notin\left[\bigcap_{i=1}^{m+1} N_{f_{i}}\right] \text {. }
$$

Лемма доказана.

Доказательство теоремы 3. Докажем необходимость. Пусть $F$ и $\phi=\left\langle\mu, \mu^{\prime}\right\rangle$ вырождены, но не согласованы. Это означает, что не выполняется по крайней мере один из трех пунктов определения.

1. Предположим, что существуют множества $A_{1}, \ldots, A_{m} \in\left\{N_{f}: f \in F\right\}$ такие, что

$$
\bigcap_{i=1}^{m} A_{i} \neq \varnothing,
$$


и точка $x^{*} \in[0,1]$ такая, что

$$
\min _{1 \leqslant i \leqslant m} \mu^{\prime}\left(A_{i}\right)\left(x^{*}\right)>\mu^{\prime}\left(\bigcap_{i=1}^{m} A_{i}\right)\left(x^{*}\right) .
$$

Для каждого $i=1, \ldots, m$ обозначим через $f_{i}$ предикат, соответствующий множеству $A_{i}$. Выберем отношение $\rho$ следующим образом:

$$
x \rho y \Longleftrightarrow\left(x \in \bigcap_{i=1}^{m} A_{i}\right) \&(y=0)
$$

Построим нечеткое отношение $\eta=\phi(\rho)$. Рассмотрим ЗИП $I=\langle[0,1],\{0\}, \rho\rangle$. Построим ИГ $U$ следующим образом: возьмем вершину, объявим ее корнем и выпустим из нее цепь, состоящую из $m$ ребер. Припишем $i$-му ребру предикат $f_{i}, i=1, \ldots, m$. Вершину, конец цепи, объявим листом и припишем ему запись 0 . По теореме 1 ИГ $U$ допустим для ЗИП $I$ над базовым множеством $F$. Рассмотрим ЗНП $\phi(I)$ и НИГ $\phi(U)$. По лемме 2 $\min _{1 \leqslant i \leqslant m} \mu^{\prime}\left(A_{i}\right)$ имеет слабый максимум, не принадлежащий $\left[\bigcap_{i=1}^{m} A_{i}\right]$. В то же время, по следствию 1 леммы 1 все слабые максимумы функции $\phi(\rho)(x, 0)$ принадлежат множеству $\left[\bigcap_{i=1}^{m} A_{i}\right]$. Следовательно,

$$
\max _{\alpha \in L_{U}(0)} \varphi_{\alpha}(x)=\min _{1 \leqslant i \leqslant m} \mu^{\prime}\left(A_{i}\right) \neq \phi(\rho)(x, 0) .
$$

Поэтому по теореме 1 НИГ $\phi(U)$ не является допустимым для ЗНП $\phi(I)$. Следовательно, базовое множество $F$ и основание $\phi=\left\langle\mu, \mu^{\prime}\right\rangle$ не вырождены. Получили противоречие.

2. Предположим, что не выполняется пункт 2 определения согласованности. Возможны следующие случаи.

a) Пусть существует $x^{*} \in\left(-b_{F}^{\prime}, b_{F}^{\prime \prime}\right)$ такой, что $\mu\left(x^{*}, 0\right) \neq \mu^{\prime}\left(x^{*}\right)$. Для определенности будем считать, что $x^{*}>0$ (случай $x^{*}<0$ рассматривается аналогично). Можно считать, что $x^{*} \in(0, b)$, где $b<b_{F}^{\prime \prime}$. Для любого $b \in\left[0, b_{F}^{\prime \prime}\right)$ существуют конечный набор предикатов $F_{\lambda} \subseteq F$ и точка $x_{0} \in[0,1-b]$ такие, что для всех $x \in\left[x_{0}, x_{0}+b\right]$ справедливо равенство $g_{\lambda}(x)=\mu^{\prime}\left(x-x_{0}\right)$. Выберем $b \geqslant x^{*}$ и для соответствующего $\lambda$ занумеруем $f \in F_{\lambda}$ числами $1, \ldots, m$, так что эти элементы примут вид $f_{1}, f_{2}, \ldots, f_{m}$.

Выберем отношение $\rho$ следующим образом:

$$
x \rho y \Longleftrightarrow\left(x \in \bigcap_{f \in F_{\lambda}} N_{f}\right) \&(y=0) \text {. }
$$

Построим нечеткое отношение $\eta=\phi(\rho)$. Рассмотрим ЗИП $I=\langle[0,1],\{0\}, \rho\rangle$. Построим ИГ $U$ следующим образом. Возьмем вершину, объявим ее корнем и выпустим из нее цепь, состоящую из $m$ ребер. Для каждого $i=1, \ldots, m$ припишем $i$-му ребру предикат $f_{i}$. Вершину, конец цепи, объявим листом и припишем ей запись 0 . По теореме 1 ИГ $U$ допустим для ЗИП $I$ над базовым множеством $F$. Рассмотрим ЗНП $\phi(I)$ и НИГ $\phi(U)$. Понятно, что

$$
\max _{\alpha \in L_{U}(0)} \varphi_{\alpha}\left(x_{0}+x^{*}\right)=\min _{1 \leqslant i \leqslant m} \phi\left(f_{i}\right)\left(x_{0}+x^{*}\right)=g_{\lambda}\left(x_{0}+x^{*}\right)=\mu^{\prime}\left(x^{*}\right) .
$$

Так как в силу пункта 1

$$
g_{\lambda}(x) \equiv \mu^{\prime}\left(\bigcap_{i=1}^{m} N_{f_{i}}\right)(x)
$$


и $g_{\lambda}\left(x_{0}\right)=1$, то вследствие леммы 1

$$
x_{0} \in\left[\bigcap_{i=1}^{m} N_{f_{i}}\right] \text {, }
$$

следовательно, $\left(x_{0}, 0\right) \in[\rho]$ и $\eta\left(x_{0}+x^{*}, 0\right) \geqslant \mu\left(x^{*}, 0\right)$.

Если $\mu\left(x^{*}, 0\right)>\mu^{\prime}\left(x^{*}\right)$, то

$$
\eta\left(x_{0}+x^{*}, 0\right) \geqslant \mu\left(x^{*}, 0\right)>\mu^{\prime}\left(x^{*}\right)=\max _{\alpha \in L_{U}(0)} \varphi_{\alpha}\left(x_{0}+x^{*}\right),
$$

следовательно, по теореме 1 НИГ $\phi(U)$ не является допустимым для ЗНП $\phi(I)$. Таким образом, базовое множество $F$ и основание $\phi$ не вырождены. Получили противоречие.

Пусть $\mu\left(x^{*}, 0\right)<\mu^{\prime}\left(x^{*}\right)$. Так как базовое множество $F$ и основание $\phi$ вырождены, по теореме 1 должно выполняться равенство $\eta\left(x_{0}+x^{*}, 0\right)=\mu^{\prime}\left(x^{*}\right)$. В таком случае $\eta\left(x_{0}+x^{*}, 0\right) \neq \mu\left(x^{*}, 0\right)$. Значит, должна существовать точка

$$
x_{1} \in\left(x_{0}, 1\right] \cap\left[\bigcap_{i=1}^{m} N_{f_{i}}\right]
$$

такая, что $\eta\left(x_{0}+x^{*}, 0\right)=\mu\left(x^{*}-x_{1}, 0\right)$. Так как $g_{\lambda}(x)=\mu^{\prime}\left(x-x_{0}\right)<1$ для всех $x \in\left[x_{0}, x_{0}+b\right]$ и в силу того, что должно выполняться соотношение $\eta(x, 0) \equiv g_{\lambda}(x)$, получаем, что $x_{1}>x_{0}+b$.

Так как $\eta\left(x_{0}+x^{*}, 0\right)=\mu\left(x^{*}-x_{1}, 0\right)$, в точке $x_{0}+x^{*}$ функщия $\eta(x, 0)$ либо возрастает, либо имеет локальный минимум. В силу вырожденности $F$ и $\phi$ по теореме 1 должно выполняться соотношение $\eta(x, 0) \equiv g_{\lambda}(x)$. Но на $\left[x_{0}, x_{0}+b\right]$

$$
g_{\lambda}(x) \equiv \mu^{\prime}\left(x-x_{0}\right) .
$$

Возьмем $\delta=\left(b-x^{*}\right) / 2$. Так как $g_{\lambda}(x)$ убывает на $\left[x_{0}, x_{0}+b\right]$,

$$
g_{\lambda}\left(x_{0}+x^{*}+\delta\right)<g_{\lambda}\left(x_{0}+x^{*}\right)=\eta\left(x_{0}+x^{*}, 0\right)<\eta\left(x_{0}+x^{*}+\delta, 0\right) .
$$

Следовательно, по теореме $1, F$ и $\phi$ не могут быть вырожденными. Получили противоречие.

б) Пусть $u_{F}^{\prime \prime}=1$, и пусть для некоторого $x^{*} \in\left(0, b_{F}^{\prime \prime}\right]$ справедливо неравенство $\mu\left(x^{*}, 0\right) \neq \mu^{\prime}\left(x^{*}\right)$. Существует конечный набор предикатов $F_{\lambda} \subseteq F$ такой, что для $x_{0}=$ $1-b_{F}^{\prime \prime} g_{\lambda}(x)=\mu^{\prime}\left(x-x_{0}\right)$ для всех $x \in\left[x_{0}, 1\right]$. Построим отношение $\rho$ и нечеткое отношение $\phi(\rho)$, ЗИП $I$ и ЗНП $\phi(I)$, ИГ $U$ и НИГ $\phi(U)$ так, как было описано выше. Необходимость равенства $\mu(x, 0)=\mu^{\prime}(x)$ для всех $x \in\left[0, b_{F}^{\prime \prime}\right)$ вытекает из рассмотрения случая а). Очевидно,

$$
x_{0}=\sup _{x \in \bigcap_{f \in F_{\lambda}} N_{f}} x
$$

поэтому $\eta(1,0)=\mu\left(1-x_{0}, 0\right)=\mu\left(b_{F}^{\prime \prime}, 0\right)$. Если $\mu\left(b_{F}^{\prime \prime}, 0\right) \neq \mu^{\prime}\left(b_{F}^{\prime \prime}\right)$, то

$$
\eta(1,0) \neq g_{\lambda}(1) \equiv \max _{\alpha \in L_{U}(0)} \varphi_{\alpha}(1),
$$

следовательно, по теореме 1 НИГ $U$ не допустим для ЗНП $\phi(I)$. Таким образом, $F$ и $\phi$ не вырождены. Получили противоречие. 
Следовательно, при $u_{F}^{\prime \prime}=1$ должно выполняться соотношение $\mu(x, 0) \equiv \mu^{\prime}(x)$ при $x \in\left[0, b_{F}^{\prime \prime}\right]$.

в) Аналогично доказывастся, что при $u_{F}^{\prime}=1$ должно выполняться соотношение $\mu(x, 0) \equiv \mu^{\prime}(x)$ при $x \in\left[-b_{F}^{\prime}, 0\right]$.

г) Пусть $u_{F}^{\prime}=u_{F}^{\prime \prime}=0$ и $u_{F}=1$. Пусть $\mu\left(x^{*}, 0\right) \neq \mu^{\prime}\left(x^{*}\right)$ для некоторого $x^{*} \in\left(0, b_{F}^{\prime \prime}\right)$ (случай $x^{*} \in\left(-b_{F}^{\prime}, 0\right)$ рассматривается аналогично). Существует конечный набор предикатов $F_{\lambda} \subseteq F$ такой, что для $x_{0}=1-b_{F}^{\prime \prime}$ справедливо равенство $g_{\lambda}(x)=\mu^{\prime}\left(x-x_{0}\right)$ для всех $x \in\left[x_{0}, 1\right]$. Построим отношение $\rho$ и нечеткое отношение $\phi(\rho)$, ЗИП $I$ и ЗНП $\phi(I)$, ИГ $U$ и НИГ $\phi(U)$ так, как было описано выше. Необходимость равенства $\mu(x, 0)=\mu^{\prime}(x)$ для всех $x \in\left[0, b_{F}^{\prime \prime}\right)$ вытекает из рассмотрения случая а). Так как в силу пункта $1 g_{\lambda}(x) \equiv \mu^{\prime}\left(\bigcap_{f \in F_{\lambda}} N_{f}\right)$ и $u_{F}^{\prime}=u_{F}^{\prime \prime}=0$, существует точка $x_{1} \in\left(x_{0}, 1\right]$ такая, что при $x \in\left(x_{0}+b_{F}^{\prime \prime}, x_{1}\right]$ справедливо соотношение $g_{\lambda}(x) \equiv \mu^{\prime}\left(x-x_{1}\right)$. Понятно, что $x_{1}=x_{0}+b_{F}^{\prime}+b_{F}^{\prime \prime}$ (при $x_{1}<x_{2}$ справедливо равенство $\left.\sup _{\mu^{\prime}\left(x-x_{1}\right)>\mu^{\prime}\left(x-x_{2}\right)} x-\sup _{\mu^{\prime}\left(x-x_{1}\right)<\mu^{\prime}\left(x-x_{2}\right)} x=x_{2}-x_{1}\right)$. Следовательно,

$$
g_{\lambda}\left(x_{0}+b_{F}^{\prime \prime}\right)=\max \left\{\mu^{\prime}\left(-b_{F}^{\prime}\right), \mu^{\prime}\left(b_{F}^{\prime \prime}\right)\right\} .
$$

Понятно, что существует точка $x_{2} \in\left[x_{0}, x_{1}\right]$ такая, что $\eta(x, 0) \equiv \mu\left(x-x_{0}, 0\right)$ при $x \in$ $\left[x_{0}, x_{2}\right)$, а при $x \in\left(x_{2}, x_{1}\right]$ справедливо соотношение $\eta(x, 0) \equiv \mu\left(x-x_{1}, 0\right)$. Необходимость равенства $\mu(x, 0)=\mu^{\prime}(x)$ для всех $x \in\left(-b_{F}^{\prime}, 0\right]$ вытекает из рассмотрения случая а). Следовательно, $x_{2}=x_{0}+b_{F}^{\prime \prime}$ и

$$
\eta\left(x_{0}+b_{F}^{\prime \prime}, 0\right)=\max \left\{\mu\left(-b_{F}^{\prime}, 0\right), \mu\left(b_{F}^{\prime \prime}, 0\right)\right\} .
$$

Если $\max \left\{\mu\left(-b_{F}^{\prime}, 0\right), \mu\left(b_{F}^{\prime \prime}, 0\right)\right\} \neq \max \left\{\mu^{\prime}\left(-b_{F}^{\prime}\right), \mu^{\prime}\left(b_{F}^{\prime \prime}\right)\right\}$, то

$$
\eta\left(x_{0}+b_{F}^{\prime \prime}, 0\right) \neq g_{\lambda}\left(x_{0}+b_{F}^{\prime \prime}\right) \equiv \max _{\alpha \in L_{U}(0)} \varphi_{\alpha}\left(x_{0}+b_{F}^{\prime \prime}\right),
$$

следовательно, по теореме 1 НИГ $U$ не допустим для ЗНП $\phi(I)$. Следовательно, $F$ и $\phi$ не вырождены. Получили противоречие. Таким образом, при $u_{F}^{\prime}=u_{F}^{\prime \prime}=0, \quad u_{F}=1$ должно выполняться соотношение $\mu(x, 0) \equiv \mu^{\prime}(x)$ при $x \in\left(-b_{F}^{\prime}, b_{F}^{\prime \prime}\right)$ и

$$
\max \left\{\mu\left(-b_{F}^{\prime}, 0\right), \mu\left(b_{F}^{\prime \prime}, 0\right)\right\}=\max \left\{\mu^{\prime}\left(-b_{F}^{\prime}\right), \mu^{\prime}\left(b_{F}^{\prime \prime}\right)\right\}
$$

д) Пусть $u_{F}^{\prime}=u_{F}^{\prime \prime}=u_{F}=0$, и пусть $\mu\left(x^{*}, 0\right) \neq \mu^{\prime}\left(x^{*}\right)$ для некоторого $x^{*} \epsilon$ $\left(0, b_{F}^{\prime \prime}\right)$ (случай $x^{*} \in\left(-b_{F}^{\prime}, 0\right)$ рассматривается аналогично). По определению $b_{F}^{\prime \prime}$ для любого $b \in\left[0, b_{F}^{\prime \prime}\right)$ должен существовать конечный набор предикатов $F_{\lambda} \subseteq F$ и $x_{0} \in$ $[0,1]$ такой, что $x_{0}+b \leqslant 1$, и $g_{\lambda}(x)=\mu^{\prime}\left(x-x_{0}\right)$ для всех $x \in\left[x_{0}, x_{0}+b\right]$. Поэтому из рассмотрения случая а) следует необходимость условия $\mu(x, 0) \equiv \mu^{\prime}(x)$ при $x \in\left(-b_{F}^{\prime}, b_{F}^{\prime \prime}\right)$.

3. Пусть не выполняется пункт 3 определения согласованности. Предположим, что существует $y_{0} \neq 0$ такой, что для некоторого $x_{0} \in[-1,0]$ справедливо неравенство $\mu\left(x_{0}, y_{0}\right)>\varepsilon_{F}^{\prime}$ (случай, когда существует $y_{0} \neq 0$ такой, что для некоторого $x_{0} \in[0,1]$ справедливо неравенство $\mu\left(x_{0}, y_{0}\right)>\varepsilon_{F}^{\prime \prime}$ рассматривается аналогично). Будем считать, что $y_{0}<0$ (случай $y_{0}>0$ рассматривается аналогично). По определению $\varepsilon_{F}^{\prime}$ найдется $\lambda$ и $v_{i}^{\lambda}$ такие, что $c_{i}^{\lambda}<\mu\left(x_{0}, y_{0}\right)$. Подберем $t \in(0,1]$ так, что $v_{i}^{\lambda}-t x_{0} \leqslant 1$. Занумеруем предикаты из $F_{\lambda}$ числами $1, \ldots, m$, так что они принимают вид $f_{1}, \ldots, f_{m}$. Выберем отношение $\rho$ следующим образом:

$$
x \rho y \Longleftrightarrow\left(\left(x \in \bigcap_{f \in F_{\lambda}} N_{f}\right) \&(y=0)\right) \vee\left(\left(x=v_{i}^{\lambda}-t x_{0}\right) \&\left(y=-t y_{0}\right)\right) \text {. }
$$


Рассмотрим ЗИП $I=\langle[0,1],\{0\}, \rho\rangle$. Построим ИГ $U$ следующим образом. Возьмем вершину, объявим ее корнем и выпустим из нее цепь, состоящую из $m$ ребер, $i$-му ребру припишем предикат $f_{i}$. Вершину, являюшуюся концом цепи, объявим листом и припишем ей запись 0 . По теореме 1 ИГ $U$ допустим для ЗИП $I$ над базовым множеством $F$. Построим нечеткое отношение $\eta=\phi(\rho)$ и рассмотрим задачу $\phi(I)$ и НИГ $\phi(U)$. Справедливы равенства

$$
\max _{\alpha \in L_{U}(0)} \varphi_{\alpha}\left(v_{i}^{\lambda}\right)=\min _{1 \leqslant i \leqslant m} \phi\left(f_{i}\right)\left(v_{i}^{\lambda}\right)=g_{\lambda}\left(v_{i}^{\lambda}\right)=c_{i}^{\lambda} .
$$

Но согласно пункту 1

$$
\eta\left(v_{i}^{\lambda}, 0\right)=\sup _{\left(x^{\prime}, y^{\prime}\right) \in \rho} \mu\left(v_{i}^{\lambda}-x^{\prime}, 0-y^{\prime}\right)=\mu\left(t x_{0}, t y_{0}\right) .
$$

Функщия $\mu$ была огределена таким образом, что при $t \in(0,1)$

$$
\mu(t x, t y) \geqslant \mu(x, y)
$$

для любых $x \in[-1,1], y \in[-1,0) \cup(0,1]$, следовательно,

$$
\mu\left(t x_{0}, t y_{0}\right) \geqslant \mu\left(x_{0}, y_{0}\right)>c_{i}^{\lambda} \text {. }
$$

Итак,

$$
\eta\left(v_{i}^{\lambda}, 0\right)>\max _{\alpha \in L_{U}(0)} \varphi_{\alpha}\left(v_{i}^{\lambda}\right)=c_{i}^{\lambda} .
$$

Поэтому по теореме 1 НИГ $\phi(U)$ не является допустимым для ЗНП $\phi(I)$. Следовательно, базовое множество $F$ и основание $\phi=\left\langle\mu, \mu^{\prime}\right\rangle$ не вырождены. Получаем противоречие.

Докажем теперь достаточность. Пусть $F$ и $\phi=\left\langle\mu, \mu^{\prime}\right\rangle$ согласованы. Возьмем произвольное отношение $\rho \in[0,1]^{2}$, произвольную ЗИП $I=\langle[0,1], V, \rho\rangle$, где $V$ - произвольное конечное подмножество $[0,1]$, и любой ИГ $U \in U^{\prime}(I, F)$. Построим нечеткое отношение $\eta=\phi(\rho)$ и перейдем к ЗНП $I^{\prime}=\phi(I)$. Рассмотрим НИГ $U^{\prime}=\phi(U)$. Рассмотрим все цепи, ведущие из корня ИГ $U$ в листья, которым приписана запись $y_{0}$. Так как $U \in \mathcal{U}^{\prime}(I, F)$, по теореме 1 множество таких листьев не пусто и

$$
\max _{\alpha \in L_{U}\left(y_{0}\right)} \varphi_{\alpha}(x) \equiv \rho\left(x, y_{0}\right)
$$

причем проводимость каждой цепи ненулевая. Если выполняется пункт 1 определения согласованности, то

$$
\min \left\{\phi\left(f_{1}\right), \ldots, \phi\left(f_{N}\right)\right\} \equiv \phi\left(f_{1} \& \ldots \& f_{N}\right)
$$

для любых предикатов $f_{1}, \ldots, f_{N} \in F$. Пусть

$$
\rho\left(x, y_{0}\right) \equiv \max _{\alpha \in L_{U}\left(y_{0}\right)} \varphi_{\alpha}(x) \equiv \max _{1 \leqslant i \leqslant M} \min _{1 \leqslant j \leqslant N_{i}} f_{i j} .
$$

Введем отношения $\rho_{i}, i=1, \ldots, M$, следующим образом:

$$
x \rho_{i} y \Longleftrightarrow\left(\left(x \in \bigcap_{j=1}^{N_{i}} N_{f_{i j}}\right) \&\left(y=y_{0}\right) \vee\left(\begin{array}{lll}
x & y
\end{array}\right) \&\left(y \neq y_{0}\right)\right) \text {. }
$$


Для произвольного отношения $\rho^{\prime}$ и произвольного $y^{\prime} \in[0,1]$ рассмотрим отношение $\pi_{\rho^{\prime} y^{\prime}}$ такое, что

$$
x \pi_{\rho^{\prime} y^{\prime}} y \Longleftrightarrow\left(x \rho^{\prime} y\right) \&\left(y=y^{\prime}\right) .
$$

Выполнение пункта 3 определения согласованности означает, что

$$
\phi\left(\rho_{i}\right)\left(x, y_{0}\right) \equiv \phi\left(\pi_{\rho_{i} y_{0}}\right)\left(x, y_{0}\right), \quad i=1, \ldots, M .
$$

Наконец, выполнение пунктов 1 и 2 определения согласованности означает, что

$$
\phi\left(\pi_{\rho_{i} y_{0}}\right)\left(x, y_{0}\right) \equiv \min _{1 \leqslant j \leqslant N_{i}} \phi\left(f_{i j}\right), \quad i=1, \ldots, M .
$$

Объединяя все три условия, получаем, что

$$
\begin{aligned}
\eta\left(x, y_{0}\right) & \equiv \phi(\rho)\left(x, y_{0}\right) \equiv \phi\left(\max _{1 \leqslant i \leqslant M} \rho_{i}\right) \equiv \max _{1 \leqslant i \leqslant M} \phi\left(\rho_{i}\right) \\
& \equiv \max _{1 \leqslant i \leqslant M} \phi\left(\pi_{\rho_{i} y_{0}}\right)\left(x, y_{0}\right) \equiv \max _{1 \leqslant i \leqslant M} \min _{1 \leqslant j \leqslant N_{i}} \phi\left(f_{i j}\right)(x) \equiv \max _{\alpha \in L_{U^{\prime}}\left(y_{0}\right)} \varphi_{\alpha}(x) .
\end{aligned}
$$

Итак, для ЗНП $I^{\prime}$ и НИГ $U^{\prime}$ выполнены условия теоремы 1 . Поэтому НИГ $U^{\prime}$ допустим для ЗНП $I^{\prime}$ над базовым множеством $F$. Произвольность выбора отношения $\rho$, ЗИП $I$ и ИГ $U$, допустимого для $I$, доказывает достаточность.

Теорема доказана.

\section{Список литературы}

1. Гасанов Э. Э, Информационно-графовая модель хранения и поиска данных. Интеллектуальные системы (1998) 3, № 3-4, 163-192.

2. Гасанов Э. Э., Об одной математической модели информационного поиска. Дискретная математика (1991) 3, № 2, 69-76.

3. Колмогоров А. Н., Фомин С. В., Элементы теории функций и функционального анализа. Наука, M., 1968. 